\title{
Hyers-Ulam-Rassias Stability of Orthogonal Quadratic Functional Equation
}

\author{
Manoj Kumar ${ }^{1}$, Renu Chugh ${ }^{2}$, Ashish $^{3}$ \\ ${ }^{1}$ Assistant Professor, ${ }^{2}$ Professor, ${ }^{3}$ Research Scholar \\ Department of Mathematics \\ Maharshi Dayanand University, Rohtak \\ 124001, INDIA
}

\begin{abstract}
In this paper, we study the Hyers-Ulam-Rassias stability of the quadratic functional equations $f(3 x \pm y)=f(x \pm y)+16 f(x)$ for the mapping $f$ from orthogonal linear space in to Banach space. Furthermore, we establish the asymptotic behavior of the above quadratic functional equation. The main result has been supported by well constructed example.
\end{abstract}

Keywords: Hyers-Ulam-Rassias stability, Orthogonal spaces, Quadratic functional equations

\section{INTRODUCTION}

In 1940, a famous talk presented by Stanislaw M. Ulam [20], triggered the study of stability problem for various functional equations. Ulam presented a number of important unsolved problems. One of the interesting problem in the theory of nonlinear analysis concerning the stability of homomorphism was as follows:

Let $G_{1}$ be a group and let $G_{2}$ be a metric group with the metric $d(.,$.$) . Given \varepsilon>0$, does there exist a $\delta>0$ such that if a mapping $h: G_{1} \rightarrow G_{2}$ satisfies the inequality $d(h(x y), h(x) h(y))<\delta$, for all $x, y \in G_{1}$, then there is a homomorphism $H: G_{1} \rightarrow G_{2}$ with $d(h(x), H(x))<\varepsilon$, for all $x \in G_{1}$ ? If the answer is affirmative, we would say that equation of homomorphism $H(x y)=H(x) H(y)$ is stable.

In 1941, D. H. Hyers [2] was the first mathematician to present the result concerning the stability of functional equations on Banach spaces. This result of Hyers [2] is stated as follows: Let $f: X \rightarrow Y$ satisfies $\|f(x+y)-f(x)-f(y)\| \leq \varepsilon$ for all $x, y \in X$ and $\varepsilon \geq 0$. Then there exists a unique additive mapping $T: X \rightarrow Y$ such that $\|f(x)-T(x)\| \leq \varepsilon$, for all $x \in X$. The generalized version of $\mathrm{D}$. H. Hyers [2] result was given by famous Greece mathematician Th. M. Rassias [23] in 1978, where $f: X \rightarrow Y$ satisfies the inequality $\|f(x+y)-f(x)-f(y)\| \leq \theta\left(\|x\|^{p}+\|y\|^{p}\right)$ for all $\quad x, y \in X$, for some $\theta \geq 0$ and $0 \leq p<1$. The stability paper [24] given by Th. M. Rassias has significantly influenced in the development of stability of functional equations and hence named as Hyers-Ulam-Rassias stability of functional equations. Further, in 1994, P. Gavruta [16] provided a further generalization in which he replaced the bound $\theta\left(\|x\|^{p}+\|y\|^{p}\right)$ by a general function $\phi(x, y)$ for the existence of unique linear mapping. During the last decades several stability results have been introduced by many famous mathematicians one may also refer to $[4,15,18,22]$.

In 1975, the orthogonally additive functional equation $f(x+y)=f(x)+f(y), x \perp y$, where $\perp$ is the orthogonality symbol was investigated by S. Gudder and D. Strawther [21]. Later on, Ger and Sikorska [19] established the orthogonal stability of above additive functional equation in the sense of J. Ratz [8] for the mapping $f: X \rightarrow Y$, where $X$ is orthogonal linear space and $Y$ is a Banach space. This result was also generalized by M.S. Moslehian [10] in the framework of Banach modules.

The orthogonally quadratic functional equation $f(x+y)+f(x-y)=2 f(x)+2 f(y)$, where $x \perp y$ and $\perp$ means the Hilbert space orthogonality was first investigated by F. Vajzovic [5]. Later on, the result of Vajzovic [5] was generalized by F. Drljevic [3], M. Fochi [12] and Gy. Szabo [7]. For further detailed study of stability of orthogonal functional equations one may also refer to $[9,11,13,14]$. The functional equation

$$
D(f(x, y))=f(3 x \pm y)-f(x \pm y)-16 f(x)
$$

is called as quadratic functional equation. In 2008, W. Towanlong and P. Nakmahachalasiant [25] established the general solution and proved the Hyers-Ulam-Rassias stability of the quadratic functional equation (1.1). In this paper, we investigate the orthogonal stability of the quadratic functional equation (1.1). Furthermore, the results have been supported by a well constructed example.

There are several orthogonality concepts on a arbitrary real normed space given by many famous mathematicians such as G. Birkhoff [6], R. C. James [17], C. R. Diminnie [1], Gy. Szabo [7], J. Ratz [8] etc. Here, we recall the orthogonality in the sense of J. Ratz [8]. In 1985, J. Ratz presented the following definition of orthogonality:

Definition 1.1. [8] Suppose $X$ is a real vector space with $\operatorname{dim} \geq 2$ and $\perp$ is a binary relation on $X$ with the following properties.

$\left(\mathrm{O}_{1}\right)$ totality of $\perp$ for zero: $x \perp 0,0 \perp x$ for all $x \in X$.

$\left(\mathrm{O}_{2}\right)$ independence: if $x, y \in X-\{0\}, x \perp y$, then $x, y$ are

linearly independent. 
$\left(\mathrm{O}_{3}\right)$ homogeneity: if $x, y \in X, x \perp y$, then $\alpha x \perp \beta y$ for

$$
\alpha, \beta \in \square \text {. }
$$

$\left(\mathrm{O}_{4}\right)$ the Thalesian property: if $P$ is a 2- dimensional subspace of

$X, x \in P$ and $\lambda \in \square^{+}$, then there exists $y_{0} \in P$ such that

$x \perp y_{0}$ and $x+y_{0} \perp \lambda x-y_{0}$

The pair $(X, \perp)$ is called an orthogonality space. By an orthogonality space we mean an orthogonality space equipped with a norm. The relation $\perp$ is called symmetric if $x \perp y$ and $y \perp x$ for all $x, y \in X$.

Definition 1.2. Let $X$ be an orthogonality normed space and $Y$ be a real Banach space. A mapping $f: X \rightarrow Y$ is said to orthogonally quadratic if it satisfies the so-called orthogonally quadratic functional equation (1.1) for all $x, y \in X$ with $x \perp y$.

\section{MAIN RESULTS}

Throughout, this section, let $(X, \perp)$ denotes an orthogonality normed space with norm $\|\cdot\|_{X}$ and $\left(Y,\|\cdot\|_{Y}\right)$ is a Banach space.

Theorem 2.1. Let $\varepsilon$ and $p(p<2)$ be non-negative real numbers. Suppose that $f: X \rightarrow Y$ is a quadratic mapping satisfying the inequality

$$
\|D(f(x, y))\|_{Y} \leq \varepsilon\left(\|x\|_{X}^{p}+\|y\|_{X}^{p}\right)
$$

for all $x, y \in X$ with $x \perp y$. Then, there exists a unique orthogonally quadratic mapping $Q: X \rightarrow Y$ such that

$$
\|f(x)-Q(x)\|_{Y} \leq \frac{\varepsilon}{2\left(3^{2}-3^{p}\right)}\|x\|_{X}^{p}
$$

for all $x \in X$.

Proof. To prove this theorem we have to show the following steps:

(i) $\left\{f\left(3^{n} x\right) / 3^{2 n}\right\}$ is a Cauchy sequence for every fixed $x \in X$ such that $x \perp 0$.

(ii) There exists a quadratic mapping $Q: X \rightarrow Y$ defined by

$$
Q(x)=\lim _{n \rightarrow \infty}\left\{f\left(3^{n} x\right) / 3^{2 n}\right\}
$$

(iii) The mapping $Q: X \rightarrow Y$ satisfies

$$
\|f(x)-Q(x)\|_{Y}<\frac{\varepsilon}{2\left(3^{2}-3^{p}\right)}\|x\|_{X}^{p} \text { for } p<2 \text {. }
$$

(iv) The mapping $Q: X \rightarrow Y$ is unique.

To prove (i) let us take $y=0$ in (2.1), we obtain

$$
\|2 f(3 x)-2 f(x)-16 f(x)\|_{Y} \leq \varepsilon\left(\|x\|_{X}^{p}+\|0\|_{X}^{p}\right)
$$

$$
\begin{aligned}
& \|2 f(3 x)-18 f(x)\|_{Y} \leq \varepsilon\left(\|x\|_{X}^{p}\right) \\
& \left\|\frac{f(3 x)}{3^{2}}-f(x)\right\|_{Y} \leq \frac{\varepsilon}{2.3^{2}}\|x\|_{X}^{p}
\end{aligned}
$$

for all $x, y \in X$ with $x \perp 0$. Now, replacing $x$ with $3 x$ and dividing by $3^{2}$ in (2.3) and then adding the resulting equation with (2.3), we obtain

$$
\left\|\frac{f\left(3^{2} x\right)}{3^{4}}-f(x)\right\|_{Y} \leq \frac{\varepsilon}{2.3^{2}}\left(1+\frac{3^{p}}{3^{2}}\right)\|x\|_{X}^{p}
$$

By using induction on $n$, we get

$$
\left\|\frac{f\left(3^{n} x\right)}{3^{2 n}}-f(x)\right\|_{Y} \leq \frac{\varepsilon}{2.3^{2}} \sum_{k=0}^{n-1} \frac{3^{p k}}{3^{2 k}}\|x\|_{X}^{p}
$$

for all $x, y \in X$ with $x \perp 0$ and $n \geq 1$. To establish that $\left\{f\left(3^{n} x\right) / 3^{2 n}\right\}$ is a Cauchy sequence. Replacing $x$ with $3^{m} x$ and dividing by $3^{2 m}$ in (2.5) we obtain for $n, m>0$.

$$
\begin{aligned}
& \left\|\frac{f\left(3^{n+m} x\right)}{3^{2 n+2 m}}-\frac{f\left(3^{m} x\right)}{3^{2 m}}\right\|_{Y} \leq \frac{\varepsilon}{2.3^{2}} \sum_{k=0}^{n-1} \frac{3^{p(k+m)}}{3^{2 k+2 m}}\|x\|_{X}^{p} \\
& \frac{1}{3^{2 m}}\left\|\frac{f\left(3^{n+m} x\right)}{3^{2 n}}-f\left(3^{m} x\right)\right\|_{Y} \leq \frac{\varepsilon}{2.3^{2} \cdot 3^{2 m-p m}} \sum_{k=0}^{n-1} \frac{3^{p k}}{3^{2 k}}\|x\|_{X}^{p}
\end{aligned}
$$

As we know for $p<2$ the right hand side of (2.6) tends to zero as $m \rightarrow \infty$ for all $x \in X$. Thus, the sequence $\left\{f\left(3^{n} x\right) / 3^{2 n}\right\}$ is convergent in $Y$. Since $Y$ is complete normed space, whence the sequence $\left\{f\left(3^{n} x\right) / 3^{2 n}\right\}$ is a Cauchy sequence for every $x \in X$. Then, there exists a orthogonally quadratic mapping $Q: X \rightarrow Y$ such that

$$
Q(x)=\lim _{n \rightarrow \infty}\left\{f\left(3^{n} x\right) / 3^{2 n}\right\} \quad \text { for all } x \in X .
$$

(ii) Now, we claim that the mapping $Q: X \rightarrow Y$ is quadratic that is it satisfies the equation (1.1). Substituting $3^{n} x$ and $3^{n} y$ at place of $x$ and $y$ in (2.1) respectively and dividing by $3^{2 n}$, we obtain

$$
\left\|\frac{D\left(f\left(3^{n} x, 3^{m} y\right)\right)}{3^{2 n}}\right\|_{Y} \leq \frac{\varepsilon}{3^{2 n}}\left(\left\|3^{n} x\right\|_{X}^{p}+\left\|3^{n} y\right\|_{X}^{p}\right)
$$

Taking $n \rightarrow \infty$ in (2.8), we have

$$
\begin{aligned}
& \|Q(3 x \pm y)-Q(x \pm y)-16 Q(x)\|_{Y} \leq 0 \\
& Q(3 x \pm y)=Q(x \pm y)+16 Q(x)
\end{aligned}
$$

for all $x, y \in X$ with $x \perp y$. Which shows that the mapping $Q: X \rightarrow Y$ is orthogonally quadratic mapping.

(iii) By making $n \rightarrow \infty$ in the equation (2.5) we obtain as follows 


$$
\|f(x)-Q(x)\|_{Y} \leq \frac{\varepsilon}{2\left(3^{2}-3^{p}\right)}\|x\|_{X}^{p} \text { for all } x \in X .
$$

(iv) Now, to prove the uniqueness of orthogonally quadratic mapping $Q: X \rightarrow Y$. We consider another orthogonally quadratic mapping $Q^{\prime}: X \rightarrow Y$ satisfying the equation (1.1). Hence,

$$
\begin{aligned}
\left\|Q(x)-Q^{\prime}(x)\right\|_{Y} \leq & \frac{1}{3^{2 n}}\left\{\left\|Q\left(3^{n} x\right)-f\left(3^{n} x\right)\right\|_{Y}+\left\|f\left(3^{n} x\right)-Q^{\prime}\left(3^{n} x\right)\right\|_{Y}\right\} \\
\leq & \frac{\varepsilon}{\left(3^{2}-3^{p}\right) 3^{n(2-p)}}\|x\|_{X}^{p} \\
& \rightarrow 0 \text { as } n \rightarrow \infty
\end{aligned}
$$

for all $x \in X$. Which implies that $Q(x)=Q^{\prime}(x)$, that means the orthogonally quadratic mapping $Q$ is unique. This completes the proof of theorem.

Theorem 2.2. Let $\varepsilon$ and $p(p>2)$ be non-negative real numbers. Suppose that $f: X \rightarrow Y$ is a quadratic mapping satisfying the inequality (2.1) for all $x, y \in X$ with $x \perp y$. Then, there exists a unique orthogonally quadratic mapping $Q: X \rightarrow Y$ such that

$\|f(x)-Q(x)\|_{Y} \leq \frac{\varepsilon}{2\left(3^{p}-3^{2}\right)}\|x\|_{X}^{p}$ for all $x \in X$.

Proof. Replacing $x$ with $x / 3$ and multiplying by $3^{2}$ in (2.3), we obtain as follows

$$
\begin{aligned}
& \left\|f(x)-3^{2} f\left(\frac{x}{3}\right)\right\|_{Y} \leq \frac{\varepsilon}{2} \cdot\left\|\frac{x}{3}\right\|_{X}^{p} \\
& \left\|f(x)-3^{2} f\left(\frac{x}{3}\right)\right\|_{Y} \leq \frac{\varepsilon}{2.3^{p}}\|x\|_{X}^{p}
\end{aligned}
$$

for all $x \in X$ with $x \perp 0$. Again replacing $x$ with $x / 3$ and multiplying by $3^{2}$ in (2.10) and then summing the resulting equation with (2.10), we obtain

$$
\left\|f(x)-3^{4} f\left(\frac{x}{3^{2}}\right)\right\|_{Y} \leq \frac{\varepsilon}{2.3^{p}}\left(1+\frac{3^{2}}{3^{p}}\right)\|x\|_{X}^{p}
$$

By using induction on $n$, we obtain

$$
\begin{aligned}
& \left\|f(x)-3^{2 n} f\left(\frac{x}{3^{n}}\right)\right\|_{Y} \leq \frac{\varepsilon}{2.3^{p}} \sum_{k=0}^{n-1} \frac{3^{2 k}}{3^{k p}}\|x\|_{X}^{p} \\
& \leq \frac{\varepsilon}{2.3^{p}} \sum_{k=0}^{\infty} \frac{3^{2 k}}{3^{k p}}\|x\|_{X}^{p}
\end{aligned}
$$

for all $x \in X$ with $x \perp 0$ and $n \geq 1$. Now, to prove the convergence of $\left\{f\left(3^{n} x\right) / 3^{2 n}\right\}$ replacing $x$ with $x / 3^{m}$ and multiplying by $3^{2 m}$ in (2.12) we obtain for $n, m>0$. $\left\|3^{2 m} f\left(\frac{x}{3^{m}}\right)-3^{2 n+2 m} f\left(\frac{x}{3^{n+m}}\right)-\right\|_{Y} \leq \frac{\varepsilon}{2.3^{m(p-2)}} \sum_{k=0}^{\infty} \frac{3^{2 k}}{3^{p(k-1)}}\|x\|_{X}^{p}$ for $p>2$ the right hand side of (2.13) tends to zero as $m \rightarrow \infty$ for all $x \in X$. Thus, the sequence $\left\{3^{2 n} f\left(x / 3^{n}\right)\right\}$ is convergent in $Y$. Since $Y$ is complete normed space, whence the sequence $\left\{3^{2 n} f\left(x / 3^{n}\right)\right\}$ is a Cauchy sequence for every $x \in X$. Then, there exists a orthogonally quadratic mapping $Q: X \rightarrow Y$ such that

$$
Q(x)=\lim _{n \rightarrow \infty}\left\{3^{2 n} f\left(x / 3^{n}\right)\right\} \text { for all } x \in X .
$$

By making $n \rightarrow \infty$ in the equation (2.13) and using (2.14), we obtain the required result (2.9).

Further, to prove that the orthogonal quadratic mapping is unique the proof is similar to that of Theorem 2.1.

Remark 2.3. In Theorem 2.1 and Theorem 2.2 the parameter $p$ is assumed to take all values except $p=2$. If $p=2$ then the theorem are not longer valid. The following example shows that the orthogonally functional equation (2.1) has no stable solution at $p=2$.

Example 2.4. Let $\phi$ be the mapping from $\square$ into itself defined by

$$
\phi(x)=\left\{\begin{array}{l}
\mu\|x\|^{2} \text { if }\|x\|<1 \\
\mu \text { otherwise }
\end{array}\right.
$$

for some $\mu>0$. Also, let $f: \square \rightarrow \square$ be a function defined as

$$
f(x)=\sum_{n=0}^{\infty} \frac{\phi\left(3^{n} x\right)}{3^{2 n}} \text { for all } x \in \square
$$

Then, $f$ satisfies the inequality

$$
\|D(f(x, y))\| \leq \mu\left(\|x\|^{2}+\|y\|^{2}\right)
$$

for all $x, y \in \square$. But there does not exists any quadratic mapping $Q: \square \rightarrow \square$ satisfying

$$
\|f(x)-Q(x)\| \leq \mu\|x\|^{2} \text { for all } x \in \square
$$

Solution. From (2.15) and (2.16), we obtain

$$
\|f(x)\| \leq \sum_{n=0}^{\infty} \frac{\mu}{3^{2 n}}=\frac{9}{8} \mu \text { for all } x \in \square
$$

which implies that the function $f$ is bounded. First we prove that the function $f$ satisfies the inequality (2.17) under the condition of (2.15). Let us consider $0<\|x\|^{2}+\|y\|^{2}=\|x+y\|^{2}<1$. Then for a positive number $k \in \square$, we have

$$
\frac{1}{3^{2 k}} \leq\|x\|^{2}+\|y\|^{2} \leq \frac{1}{3^{2(k-1)}} \text { for all } x, y \in \square
$$

which implies that $3^{k-1}\|x\|<1,3^{k-1}\|y\|<1,3^{k-1}(\|x+y\|)<1$, $3^{k-1}(\|x-y\|)<1,3^{k-1}(\|3 x+y\|)<1,3^{k-1}(\|3 x-y\|)<1$ for all $n \in\{0,1,2, \ldots, \square-1\}$, whence 


$$
\begin{aligned}
\|D(f(x, y))\| & \leq \sum_{n=0}^{k-1} \frac{\mu\left\|3^{n}(3 x \pm y)\right\|^{2}-\mu\left\|3^{n}(x \pm y)\right\|^{2}-16 \mu\left\|3^{n} x\right\|^{2}}{3^{2 n}} \\
& \leq \sum_{n=k}^{\infty} \frac{\left\|\phi\left(3^{n}(3 x \pm y)\right)-\phi\left(3^{n}(x \pm y)\right)-16 \phi\left(3^{n} x\right)\right\|}{3^{2 n}} \\
& \leq \sum_{n=k}^{\infty} \frac{20}{9^{n}} \mu
\end{aligned}
$$

Hence $f$ satisfies inequality (2.17) for all $x, y \in \square$ with the condition $\|x\|^{2}+\|y\|^{2}<1$, Further, let $\|x\|^{2}+\|y\|^{2} \geq 1$, then

$$
\begin{aligned}
\|D(f(x, y))\| & \leq \sum_{n=0}^{\infty} \frac{\left\|\phi\left(3^{n}(3 x \pm y)\right)-\phi\left(3^{n}(x \pm y)\right)-16 \phi\left(3^{n} x\right)\right\|}{3^{2 n}} \\
& \leq \sum_{n=0}^{\infty} \frac{20}{9^{n}} \mu \leq 5 \mu\left(\|x\|^{2}+\|y\|^{2}\right)
\end{aligned}
$$

Hence, to prove the required result let us consider an orthogonally quadratic mapping $Q: \square \rightarrow \square$ and a constant number $\eta>0$ such that

$$
\|f(x)-Q(x)\| \leq \eta\|x\|^{2} \text { for all } x \in \square .
$$

Since, $f$ is bounded, $Q$ is also bounded on any open interval containing the origin zero. So, $Q$ has the form $Q(x)=c\|x\|^{2}$ for all $x \in \square$, and $c$ is constant. Therefore, we have

$$
\begin{aligned}
& \|f(x)-c\| x\left\|^{2}\right\| \leq \eta\|x\|^{2} \\
& \|f(x)\| \leq(\eta+|c|)\|x\|^{2} \text { for all } x \in \square .
\end{aligned}
$$

Also for $\square \in I^{+}$this implies $\square \mu>\eta+|c|$ and also for $x \in \square$ implies $\|x\| \in\left(0,1 / 3^{\square-1}\right), \quad$ then, $\quad 0<\left\|3^{n} x\right\|<1 \quad$ for $\quad$ all $n \in\{0,1,2, \ldots, \square-1\}$. That implies

$$
\begin{aligned}
f(x) & =\sum_{n=0}^{\infty} \frac{\phi\left(3^{n} x\right)}{3^{2 n}} \geq \sum_{n=0}^{\square-1} \frac{\phi\left(3^{n} x\right)}{3^{2 n}} \\
& =\sum_{n=0}^{-1} \frac{\mu 3^{2 n}\|x\|^{2}}{3^{2 n}} \\
& =\square \mu\|x\|^{2}>(\eta+|c|)\|x\|^{2}
\end{aligned}
$$

Which implies that orthogonally quadratic mapping $Q: \square \rightarrow \square$ not satisfies the inequality (2.18), that is, the given orthogonally quadratic functional equation is not stable at $p=2$. Which is a contradiction.

\section{CONCLUSION}

First we proved the Hyers-Ulam-Rassias stability of the quadratic functional equations (1.1) for the mapping $f$ from orthogonal linear space in to Banach space. Furthermore, we established an example showing that at $p=2$ the results are not longer valid.

\section{REFERENCES}

[1] C. R. Dimmine, R. W. Freese, E. Z. Andalafte, An extension of Pythagorean and isosceles orthogonality and a characterization of inner product spaces, J. Approx. Theory (39)(4)(1983), 295-298.

[2] D. H. Hyers, On the Stability of the Linear Functional Equation, Proc. Nat. Acad. Sci. U.S.A. (27)(1941), 222 224.

[3] F. Drljevic, On a functional which is quadratic on Aorthogonal vectors, publ. Inst. Math. (Beograd), (54)(1986), 63-71.

[4] F. Skof, Local properties and approximations of operators, Rend. Sem. Mat. Fis. Milano, (53)(1983),113129.

[5] F. Vajzovic, Uber das functional $\mathrm{H}$ mit der Eigenschaft: $(x, y)=0$ implies $H(x+y)+H(x-y)=2 H(x)+2 H(y)$, Glasnik Mat. Ser. III, (22)(1967), 73-81.

[6] G. Birkhoff, Orthogonality in linear metric spaces, Duke Math. J., (1)(1995), 169-172.

[7] Gy. Szabo, Sesqulinear-orthogonally quadratic mappings, Aequationes Math., (40)(1990), 190-200.

[8] J. Ratz, On orthogonality additive mappings, Aeq. Math. (28)(1985), 35-49.

[9] K. Sundaresan, Orthogonality and nonlinear functional on Banach spaces, Proc. Amer. Math. Soc., (34)(1972), 187-190

[10] M. S. Moslehian, On the stability of the orthogonal Pexiderized Cauchy equation, J. Math. Anl. Appl., (318)(1)(2006), 211-223.

[11] M. S. Moslehian, On the Orthogonal Stability of the Pexiderized Quadratic Equation, J. Difference Equ. Appl., (11)(2005), 999-1004.

[12] M. Fochi, Functional equations in A-orthogonal vectors, Aequationes Math., (38)(1989), 28-40.

[13] M. Mirzavaziri, M, S, Moslehian, A fixed point approach to stability of a quadratic equation, Bull. Braz. Math. Soc., (37)(3)(2006), 361-376.

[14] O. P. Kapoor, J. Prasad, Orthogonality and Characterizations of inner product spaces, Bull. Austral. Math. Soc., (19)(3)(1978), 403-416.

[15] P. W. Cholewa, Remarks on the stability of functional equations, Aequations Math. (27)(1984), 76-86.

[16] P. Gavruta, A Generalization of the Hyers-Ulam-Rassias Stability of Approximately Additive Mappings, J. Math. Anal. Appl. (184)(1994), 431-436.

[17] R. C. James, Orthogonality in normed linear spaces, Duke Math. J., (12)(1945), 291-302.

[18] R. Chugh and Ashish, On the stability of generalized Cauchy linear functional equations, Int. J. of Math. Anal., (6)(29)(2012), 1403 - 1413.

[19] R. Ger and J. Sikorska, Stability of the orthogonal additivity , Bull. Polish Acad. Sci. Math., (43)(1995),143-151. 
[20] S. M. Ulam, A Collection of the Mathematical Problems, Interscience Publ. New York, 1960.

[21] S. Gudder and D. Strawther, Orthogonality additive and Orthogonality increasing functions on vector spaces, Pacific J. Math., (58)(1975), 427-436.

[22] T. Aoki, On the stability of the linear transformation in Banach spaces, J. of Math. Soc., (2)(1-2)(1950), 64-66.

[23] Th. M. Rassias, On the stability of the Linear mapping in Banach spaces, Procc. of the Amer. Math. Soc., (72)(2)(1978), 297-300.
[24] Th.M. Rassias, On the Stability of Functional Equations in Banach spaces, J. Math. Anal. Appl., (251)(2000), 264-284.

[25] W. Towanlong, p. Nakmahachalasiant, A quadratic functional equation and its generalized Hyers-UlamRassias stability, Thai j. of Math., Special Issue (Annual meeting in Mathematics), (2008), 85-91. 